\title{
Evaluation of a Pseudoceramide Moisturizer in Patients with Mild-to-Moderate Atopic Dermatitis
}

\author{
Amelie Clementine Seghers - Sophie Chan Cai • \\ Madeleine Sheun Ling Ho • Yoke Chin Giam • Lucinda Tan • \\ Carina Marie Grönhagen • Mark Boon Yang Tang
}

To view enhanced content go to www.dermtherapy-open.com Received: January 21, 2014 / Published online: March 12, 2014

(c) The Author(s) 2014. This article is published with open access at Springerlink.com

\section{ABSTRACT}

Introduction: To evaluate the efficacy and safety of a pseudoceramide-containing moisturizer as maintenance therapy in patients with mild-to-moderate atopic dermatitis (AD).

Methods: This was a prospective, single-arm, open-label clinical trial of a twice-daily application of a pseudoceramide-containing moisturizer for 4 weeks as maintenance therapy in 40 patients with stable, mild-tomoderate $\mathrm{AD}$ in a tropical climate. Clinical and skin barrier assessment was done at week 0 , week 2 and week 4 . Any adverse effects were also recorded during the study period.

Electronic supplementary material The online version of this article (doi:10.1007/s13555-014-0048-z) contains supplementary material, which is available to authorized users.

A. C. Seghers · M. S. L. Ho · Y. C. Giam · L. Tan ·

C. M. Grönhagen · M. B. Y. Tang ( $₫)$

National Skin Center, 1 Mandalay Road,

Novena, Singapore

e-mail: marktang@nsc.gov.sg

C. M. Grönhagen

e-mail: gronhagencm@nsc.gov.sg

S. C. Cai

Duke-National University, Singapore, Singapore
Results: The objective scoring atopic dermatitis decreased from 29.1 [interquartile range (IQR) 21.9-33.7] at week 0 to 22.0 (IQR 21.2-27.8) at week $4(p<0.001)$. There was no detectable difference in transepidermal water loss after 4 weeks; however, stratum corneum (SC) hydration was significantly increased from 39.7 (IQR 35.3-46.4) at week 0 to 49.2 (IQR 41.2-54.6) after 4 weeks $(p<0.001)$. Both Dermatology Life Quality Index and patient-oriented eczema measure showed significant improvement at week 4 $(p<0.001)$. The moisturizer was well tolerated with no serious adverse events recorded.

Conclusion: After 4 weeks of barrier maintenance therapy with a pseudoceramide moisturizer, there was a significant improvement in disease severity, SC hydration and quality of life in both pediatric and adult patients with mild-to-moderate $\mathrm{AD}$.

Keywords: Atopic dermatitis; Ceramides; Dermatology; Pseudoceramide moisturizer; Skin barrier; Objective scoring atopic dermatitis (SCORAD); Stratum corneum hydration; Transepidermal water loss 


\section{INTRODUCTION}

Atopic dermatitis (AD) is a chronic, relapsing, inflammatory skin disease causing impaired quality of life. AD affects about $10-30 \%$ of children and $2-10 \%$ of adults [1-4].

Skin barrier dysfunction plays a critical role in the initiation, perpetuation and exacerbation of $\mathrm{AD}$ and the key players for a normal epidermal barrier function include lipids and proteins in the stratum corneum. Ceramides are the major constituent (50\%) of these lipids [5] and have been shown to have a high waterholding capacity [6]. Patients with AD have been shown to have lower levels of ceramides, particularly type 1 and $3[7,8]$. More recent studies have shown that in addition to the reduction of ceramides in AD skin, an increased level of short-chain ceramides can lead to an aberrant lipid organization and decreased skin barrier function in AD patients $[9,10]$. Hence, a therapeutic approach to repair the skin barrier defect in $\mathrm{AD}$ is to normalize the amount of ceramides in the skin. The use of ceramide containing topical creams has been shown to be beneficial in the management of AD [11-13]. However, additional research is needed to determine the optimal formulary compositions.

Ceramides can be extracted from natural animal sources, but this process is lengthy and expensive with limited availability. Furthermore, there are concerns about the toxicity and the risk of transmissible infections, such as bovine spongiform encephalopathy, from bovine-derived sources [14]. Hence, synthetic pseudoceramides, which have been developed and used successfully in skin barrier repair therapy $[15,16]$, have a good safety profile and are less expensive than natural ceramides $[5,14]$.

The primary objective of this study was to demonstrate the safety and efficacy of the use of a pseudoceramide-containing moisturizer in both adults and children with mild-tomoderate $\mathrm{AD}$ as maintenance therapy in a tropical climate. Secondary aims were to evaluate skin physiological measurements and also the patients' acceptability and adherence to the moisturizer.

\section{METHODS}

This was a prospective, 4-week, non-controlled open-label clinical trial to determine the safety and efficacy of a twice-daily application of a pseudoceramide moisturizer for the maintenance therapy of mild-to-moderate $\mathrm{AD}$ in both children and adults.

The test product in this study was Curel $^{\circledR}$ Moisture cream (Kao Corporation, Tokyo, Japan), which contained $8 \%$ of a synthetic pseudoceramide, sphingolipid E (cetylpropyleneglycolhydroxy-ethylpalmitamide), which is analogous to endogenous ceramide type 2. It also contained Eucalyptus globules extract that has been shown to increase endogenous production of ceramides by keratinocytes [17]. The full ingredients of the study cream are listed in Table 1. Earlier animal safety studies have shown that it is non-toxic [18-20].

\section{Study Subjects}

Forty subjects were recruited from the outpatient dermatology clinics at the National Skin Centre, Singapore. Inclusion criteria included an unequivocal diagnosis of $\mathrm{AD}$ based on the UK Working Party's Group criteria [21], age between 7-60 years old, with stable, mild-to-moderate $\mathrm{AD}$ as defined by objective SCORing Atopic Dermatitis (SCORAD) scores of $<15$ for mild $\mathrm{AD}$, and 
Table 1 Contents for Curel $^{\circledR}$ Moisture cream (Kao Corporation, Tokyo, Japan) moisture cream

Allantoin

Bis-methoxypropylamido isodocosane

Butylene glycol

Cetyl dimethicone

Cetyl-propyleneglycolhydroxy-ethylpalmitamide,

8\% synthetic pseudoceramide (Sphingolipid E)

Cyclopentasiloxane

Dextrin palmitate

Dimethicone

Dipentaerythrityl hexahydroxystearate

Eucalyptus globulus leaf extract

Glycerine

Isostearyl glyceryl ether
Magnesium sulfate

Methyl paraben

Neopentyl glycol dicaprate

PEG-5 hydrogenated castor oil

PEG-12 dimethicone

Polyglyceryl-2 Diisostearate

Sodium hydroxide

Squalane

Succinic acid

Tocopherol

Trisiloxane

Water
15-40 for moderate AD [22-24]. Exclusion criteria were a recent disease flare or the use of any systemic therapy, including antibiotics, phototherapy, corticosteroids or immunosuppressants, within the past 4 weeks.

\section{Study Design}

There were a total of three study visits, namely, week 0 , week 2 and week 4 . At each visit, study subjects underwent clinical examination (including evaluation of any adverse events), skin physiological measurements and completed questionnaires related to quality of life, adverse events and their use of the study cream. The study was conducted in Singapore, which has a tropical climate with no seasonal variation. All subjects were instructed to use the study cream at least twice a day for 4 weeks on all affected areas as well as other body areas, including the forearms and cubital fossae. Subjects were allowed to use their standard medicated creams as needed, in event of a disease flare. No other moisturizers were allowed during the study period and all subjects were provided with a standardized soap-free cleanser for bathing. The use of topical corticosteroids was documented, and the amount of the study cream used was assessed by recording the weight of the returned containers at the end of the study period.

\section{Clinical Assessment of AD Severity}

Disease severity of $\mathrm{AD}$ was assessed using the objective and total SCORAD index and the three-item-severity (TIS) scores (maximum TIS score 9) [22-24]. Each case was evaluated by the same dermatologist to minimize inter-observer variability.

\section{Skin Physiological Studies}

Standardized measurements of stratum corneum (SC) hydration and transepidermal water loss (TEWL) were performed by a single technician. Testing was performed at a fixed 
point, defined as $3 \mathrm{~cm}$ distal from the cubital crease over the flexor aspect of both forearms. Measurements were taken in triplicate for each site, the mean value being used for analysis. There was a washout period of 3 days prior to baseline measurements (visit 1), and subjects were instructed not to use any topical products on both forearms.

SC hydration was measured using a Corneometer $^{\circledR}$ CM 825 (Courage \& Khazaka $\mathrm{GmbH}$, Cologne, Germany) and expressed in arbitrary units between 0 and 130 . A value $>50$ from the arms was interpreted as sufficiently moisturized, $35-50$ as dry and $<35$ as very dry $[25,26]$. TEWL was obtained using a Tewameter $^{\circledR}$ TM 300 (Courage \& Khazaka $\mathrm{GmbH}$, Cologne, Germany) and recorded as $\mathrm{g} / \mathrm{m}^{2} / \mathrm{h}$. All measurements were performed in the same room with a controlled temperature of $25^{\circ} \mathrm{C}$, after a 15 -min acclimatization.

\section{Patient's Assessment of AD severity}

Subjective scores included the Dermatology Life Quality Index (DLQI) (maximum DLQI score 30) $[27,28]$ and the patient-oriented eczema measure (POEM) (maximum POEM score 28) $[29,30]$. Both the adult or pediatric versions of the DLQI and POEM were used accordingly. In addition, subjective scores for both pruritus and insomnia (scale from 0 to 10) were analyzed during the study period. The subject's evaluation of the study cream was also assessed at the end of the study.

\section{Statistical Analysis}

Changes in disease severity scores, skin physiological measurements and patient subjective severity scores were analyzed at week 0 , week 2 and week 4 . Intention-to-treat analysis was performed. Given that these measurements were not normally distributed, the median and interquartile range (IQR) was used as descriptive statistics and overall improvements were analyzed using Friedman test. When there was significant overall improvement, post hoc pairwise comparison between weeks was conducted using the Wilcoxon signed-rank test with Bonferroni correction to test the median for paired results. The data were analyzed using SPSS $^{\circledR}$ version 21 (SPSS Inc., Chicago, IL, USA). Differences were considered significant at $p<0.05$.

This study was approved by the institution's Domain Specific Review Board. All procedures followed were in accordance with the ethical standards of the Helsinki Declaration of 1975, as revised in 2000 and 2008. Written informed consent was obtained from all patients or their caregivers.

\section{RESULTS}

\section{Subject Characteristics}

A total of 40 subjects with mild-to-moderate AD were recruited. There were 26 adults and 14 children (Table 2). The majority were male $(75 \%, n=30)$ and Chinese $(92.5 \%, n=37)$, with a median age of 20.5 years (range 7-37). The overall median objective SCORAD at baseline was 29.1 (IQR 21.9-33.7).

\section{Clinical Assessment of AD severity}

There was an improvement in the SCORAD and TIS scores at week 2 and week 4 . At week 2 , the objective SCORAD decreased to 25.1 (IQR 19.3-29.2) and at week 4, there was a further decrease to 22.0 (IQR 21.2-27.8) (Fig. 1). The median total SCORAD showed a similar reduction during the study period (Table 3 ). 
Table 2 Demographic details and clinical characteristics of the patients

\begin{tabular}{llll}
\hline & Total $(\boldsymbol{n}=\mathbf{4 0})$ & Adults 17-60 years $(\boldsymbol{n}=\mathbf{2 6})$ & Children 7-16 years $(\boldsymbol{n}=\mathbf{1 4})$ \\
\hline Ethnicity & 37 & 25 & 12 \\
Chinese & 1 & 0 & 1 \\
Malay & 1 & 1 & 0 \\
Indian & 1 & 0 & 1 \\
Others & & & \\
Gender & 30 & 23 & 7 \\
Male & 10 & 3 & 7 \\
Female & $20.5(7-37)$ & $22(17-37)$ & $10(7-16)$ \\
Age (year) median (range) & $38.8(28.6-43.8)$ & $41.3(20.2-51.0)$ & $28.5(14.7-53.0)$ \\
Median Total SCORAD (IQR) &
\end{tabular}

IQR interquartile range, SCORAD scoring atopic dermatitis

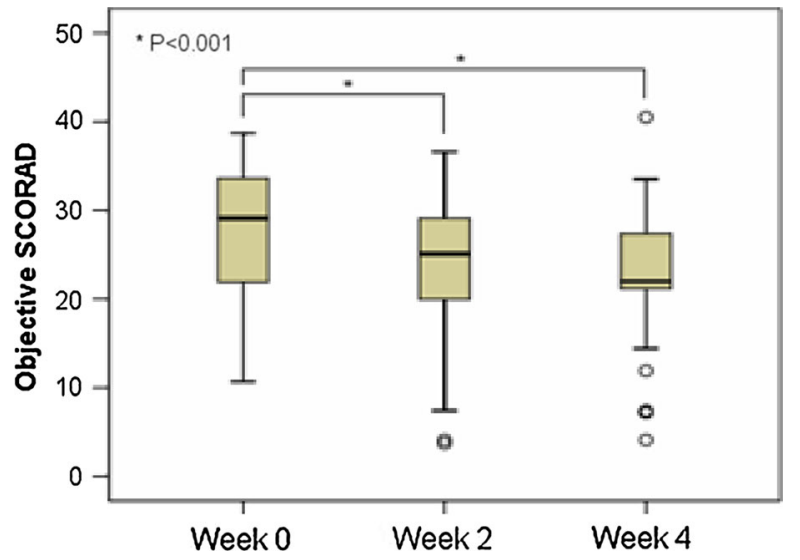

Fig. 1 Objective scoring atopic dermatitis (SCORAD) assessment shows clinical improvement after 4 weeks use of the pseudoceramide-containing moisturizer

Both overall reduction of objective SCORAD and total SCORAD were statistically significant $(p<0.001)$. The mean TIS score also decreased over 4 weeks from a baseline score of 3 (IQR 3-5) to 2 points (IQR $1-3)$ at week $4(p<0.001)$ (Table 3$)$.

\section{Skin Physiological Measurements}

SC hydration improved in 29 (85\%) of the study subjects. The median of skin hydration at baseline was 39.7 (IQR 35.3-46.4) and increased to 49.2 (IQR 41.2-54.6) at week 4 $(p<0.001)$ (Table 3).

Paradoxically, the median TEWL increased from 9.4 at baseline to 11.2 at week $4(p=0.1)$ (Table 3).

\section{Patient Subjective symptoms}

There was improvement in DLQI from a median baseline score of 6.5 (IQR 3-11) to 3 (IQR 2-7) at week $4(p<0.001)$ (Table 3$)$. The POEM showed a similar improvement: from 13 (IQR 2-7) at baseline to 6.5 (IQR $2-7)$ at week $4(p<0.001)$ (Table 3). The pruritus score was reduced from 6 (IQR 5-7) to 4 (IQR 2-6) over the study period $(p<0.001)$. A noticeable but not statistically significant improvement was seen in the insomnia score: from 2 (IQR 0-6) points on the visual analogue scale (VAS) scale to 0 (IQR $0-3)$ points at week $4(p=0.03)$.

\section{Adverse Events}

At week 2, five (12.5\%) subjects reported pruritus and one (2.5\%) patient reported 


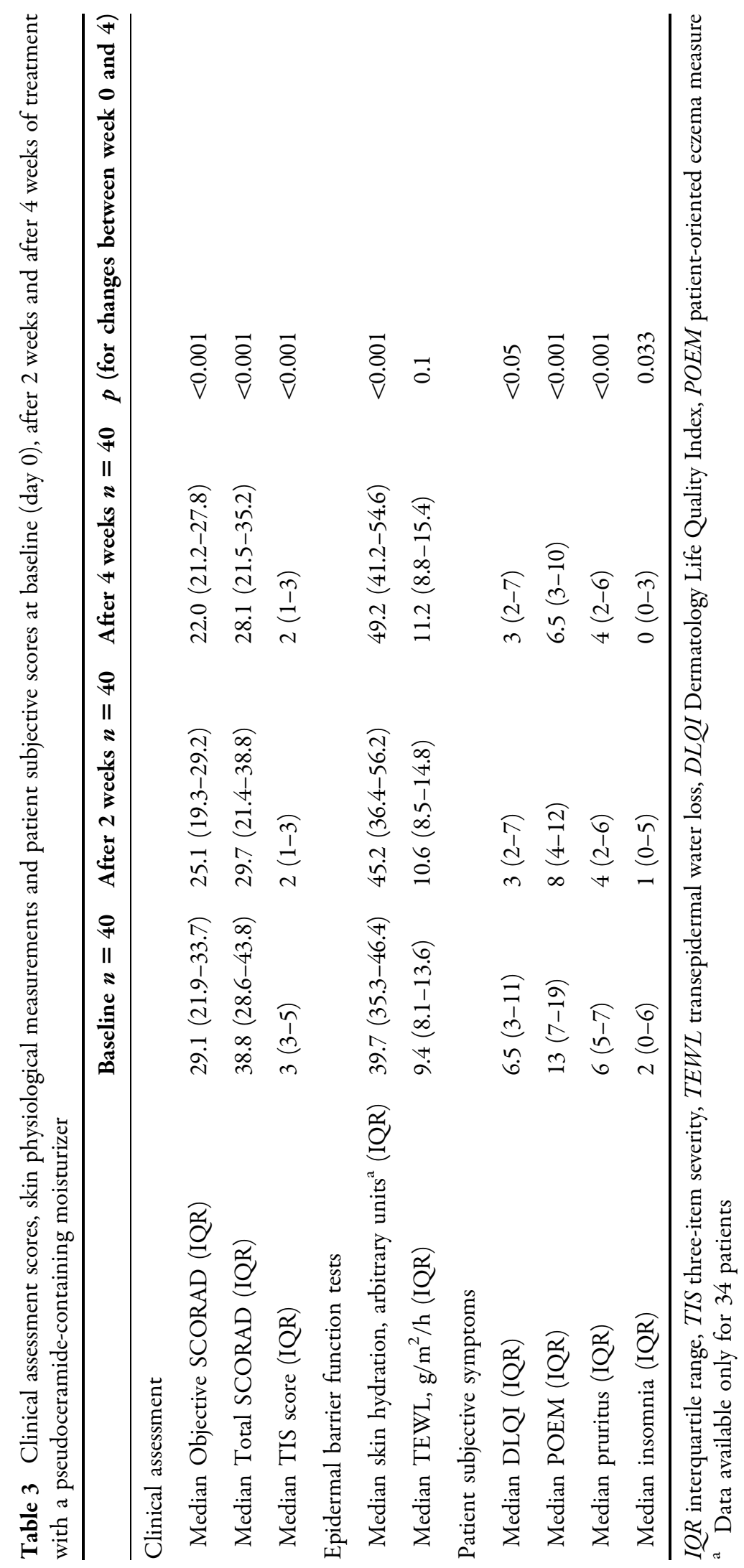


warmth sensation after application of the test cream. At week 4, 2 (5\%) patients reported pruritus after application of the test product. One patient discontinued the creams at week 3 after he developed worsening of rashes and acneiform papules on the face. There were no other serious adverse events noted.

\section{Patient Adherence and Evaluation}

At the end of the study period, 23 (57.5\%) of the subjects rated the study cream as excellent or very good, $13(32.5 \%)$ rated it as good and the remaining $4(10 \%)$ rated it as fair. The majority $(82.5 \%)$ of subjects liked the cream and reported that it improved dryness, provided symptom relief and improved skin texture.

Treatment compliance was assessed by the physician; 27 (67.5\%) patients were reported to have excellent compliance, 11 (27.5\%) moderate compliance and $2(5 \%)$ poor compliance.

There was a wide variation in the total quantity of study cream used with a mean quantity of $94 \mathrm{~g}$ (range $12-396 \mathrm{~g}, n=34$ ). There was no difference in the amount used by adults or children. No correlation between the quantity of cream used and the improvement in objective SCORAD during the study period was found.

Prior to study inclusion, all participants used topical corticosteroids. At the final follow-up, 5 $(12.5 \%)$ subjects had discontinued the use of topical corticosteroids.

\section{DISCUSSION}

This prospective open-label clinical trial found that the use of a pseudoceramide moisturizer was effective in improving disease severity, stratum corneum hydration, itch scores and quality of life indices in both pediatric and adult patients with mild or moderate AD. After a 4-week use of the pseudoceramide moisturizer, there was improvement in objective and total SCORAD, TIS, DLQI and POEM scores, which was statistically significant. This is in agreement with previous studies which showed that the use of "physiologic" ceramide moisturizers was effective in improving disease severity, decreasing steroid use and preventing disease flares $[11,12,31]$. This underscores the importance of long-term skin barrier repair in the maintenance therapy of $\mathrm{AD}[32,33]$.

While SC hydration improved significantly, it was interesting that the authors did not find any significant difference for TEWL measurements. Standardization of TEWL measurements can be technically difficult and similar paradoxical findings have been reported in other moisturizer studies [13, 31, 34, 35]. Furthermore, SC hydration is a more sensitive measure of the skin barrier function than TEWL in AD patients [36], as the relationship between TEWL and skin dryness is complex, whereby changes in dryness do not necessarily reflect simultaneous changes in TEWL [37].

The pseudoceramide moisturizer was safe and well tolerated with only $5 \%$ of patients reporting pruritus after application. Only 1 patient discontinued with the moisturizer shortly before the end of the study because of a facial rash. It is well established that topical applications, including placebo creams, can cause a transient itch or stinging [38, 39]. In general, the cream was very well tolerated and the majority of patients had positive opinions regarding the efficacy and cosmetic acceptability of the creams. On average, $94 \mathrm{~g}$ of the study cream was used during the whole study period. This may be considered low, but it is noted that subjects were only provided with one $72 \mathrm{~g}$ tube of study cream every 2 weeks. Despite a smaller body surface area, children were found to have used the same amount of study cream as adult subjects. 
This study had several limitations, namely, the small study size, non-blinded design, short duration and the lack of a control arm. The authors also did not obtain information on the exact amount of pseudoceramide moisturizer or topical corticosteroids used over specific affected areas.

\section{CONCLUSION}

This study showed that the regular use of a pseudoceramide-containing moisturizer was effective and safe as maintenance therapy in mild-to-moderate $\mathrm{AD}$ patients. Further additional, larger comparative trials against placebo creams or "standard" non-lipid-based moisturizers would be needed to further clarify the exact contributory role of lipid-based moisturizers in $\mathrm{AD}$ therapy.

\section{ACKNOWLEDGMENTS}

The authors would like to thank Ms. Karlina Tjao and Ms. Virlynn Tan Wei Ding of the National Skin Center (Novena, Singapore) for their thoughtful assistance with the statistical analysis. All named authors meet the ICMJE criteria for authorship for this manuscript, take responsibility for the integrity of the work as a whole, and have given final approval for the version to be published.

Sponsorship and article processing charges for this study was funded by Kao Corporation (Tokyo, Japan).

Conflict of interest. AC Seghers, SC Cai, MSL Ho, YC Giam, L Tan, CM Grönhagen and MBY Tang declare no conflicts of interest.

Compliance with ethics. This study was approved by the institution's Domain Specific Review Board. All procedures followed were in accordance with the ethical standards of the Helsinki Declaration of 1975, as revised in 2000 and 2008. Written informed consent was obtained from all patients or their caregivers.

Open Access. This article is distributed under the terms of the Creative Commons Attribution Noncommercial License which permits any noncommercial use, distribution, and reproduction in any medium, provided the original author(s) and the source are credited.

\section{REFERENCES}

1. Kim HY, Kwon EB, Baek JH, Shin YH, Yum HY, Jee $\mathrm{HM}$, et al. Prevalence and comorbidity of allergic diseases in preschool children. Korean J Pediatr. 2013;56:338-42.

2. Leung DY, Bieber T. Atopic dermatitis. Lancet. 2003;361:151-60.

3. Bieber T. Atopic dermatitis. $\mathrm{N}$ Engl J Med. 2008;358:1483-94.

4. Silverberg JI, Hanifin JM. Adult eczema prevalence and associations with asthma and other health and demographic factors: a US population-based study. J Allergy Clin Immunol. 2013;132:1132-8.

5. Sajic D, Asiniwasis R, Skotnicki-Grant S. A look at epidermal barrier function in atopic dermatitis: physiologic lipid replacement and the role of ceramides. Skin Ther Lett. 2012;17(7):6-9.

6. Imokawa G, Akasaki S, Hattori M, Yoshizuka N. Selective recovery of deranged water-holding properties by stratum corneum lipids. J Invest Dermatol. 1986;87(6):758-61.

7. Imokawa G, Abe A, Jin K, Higaki Y, Kawashima M, Hidano A. Decreased level of ceramides in stratum corneum of atopic dermatitis: an etiologic factor in atopic dry skin? J Invest Dermatol. 1991;96(4):523-6.

8. Jungersted JM, Hellgren LI, Jemec GB, Agner T. Lipids and skin barrier function-a clinical perspective. Contact Dermat. 2008;58(5):255-62.

9. Bolognia JL, Jorizzo JJ, Schaffer JV, editors. Dermatology, 3rd edn. 2012. 
10. Janssens $M$, van Smeden J, Gooris GS, Bras W, Portale G, Caspers PJ, et al. Increase in short-chain ceramides correlates with an altered lipid organization and decreased barrier function in atopic eczema patients. J Lipid Res. 2012;53(12): $2755-66$.

11. Simpson E, Bohling A, Bielfeldt S, Bosc C, Kerrouche $\mathrm{N}$. Improvement of skin barrier function in atopic dermatitis patients with a new moisturizer containing a ceramide precursor. J Dermatol Treat. 2013;24(2):122-5.

12. Jungersted JM, Agner T. Eczema and ceramides: an update. Contact Dermat. 2013;69(2):65-71.

13. Hon KL, Wang SS, Lau Z, Lee HC, Lee KK, Leung TF, et al. Pseudoceramide for childhood eczema: does it work? Hong Kong Med J Xianggang yi xue za zhi/ Hong Kong Acad Med. 2011;17(2):132-6.

14. Uchida Y, Holleran WM, Elias PM. On the effects of topical synthetic pseudoceramides: comparison of possible keratinocyte toxicities provoked by the pseudoceramides, PC104 and BIO391, and natural ceramides. J Dermatol Sci. 2008;51(1):37-43.

15. Takagi Y, Nakagawa H, Higuchi K, Imokawa G. Characterization of surfactant-induced skin damage through barrier recovery induced by pseudoacylceramides. Dermatology. 2005;211(2): 128-34.

16. Vavrova K, Hrabalek A, Mac-Mary S, Humbert $P$, Muret P. Ceramide analogue $14 \mathrm{~S} 24$ selectively recovers perturbed human skin barrier. $\mathrm{Br} \mathrm{J}$ Dermatol. 2007;157(4):704-12.

17. Ishikawa J, Shimotoyodome Y, Chen S, Ohkubo K, Takagi Y, Fujimura T, et al. Eucalyptus increases ceramide levels in keratinocytes and improves stratum corneum function. Int $\mathrm{J}$ Cosmet Sci. 2012;34(1):17-22.

18. Morita O, Ogura R, Hayashi K, Okuda M, Yoshimura K. Safety studies of pseudo-ceramide SLE66: acute and short-term toxicity. Food Chem Toxicol: Int $\mathrm{J}$ Publ $\mathrm{Br}$ Ind Biol Res Assoc. 2009;47(4):669-73.

19. Morita O, Ogura R, Hayashi K, Okuda M, Yoshimura K. Safety studies of Pseudo-Ceramide SLE66. Part 2: metabolism, cytotoxicity and genotoxicity. Food Chem Toxicol: Int J Publ Br Ind Biol Res Assoc. 2009.

20. Morita O, Knapp JF, Tamaki Y, Stump DG, Nemec MD, Yoshimura K. Safety studies of pseudoceramide SLE66. Part 3: effects on embryo/fetal development in rats. Food Chem Toxicol: Int J Publ Br Ind Biol Res Assoc. 2009;47(4):681-6.
21. Williams HC, Burney PG, Pembroke AC, Hay RJ. The U.K. working party's diagnostic criteria for atopic dermatitis. III. Independent hospital validation. Br J Dermatol. 1994;131(3):406-16.

22. Severity scoring of atopic dermatitis: the SCORAD index. Consensus Report of the European Task Force on Atopic Dermatitis. Dermatology 1993;186(1):23-31.

23. Charman C, Chambers C, Williams H. Measuring atopic dermatitis severity in randomized controlled clinical trials: what exactly are we measuring? J Invest Dermatol. 2003;120(6):932-41.

24. Oranje AP, Glazenburg EJ, Wolkerstorfer A, de Waard-van der Spek FB. Practical issues on interpretation of scoring atopic dermatitis: the SCORAD index, objective SCORAD and the threeitem severity score. Br J Dermatol. 2007;157(4): $645-8$.

25. GmbH CE. Information and Operating Instructions for the Corneometer CM825. http:// www.courage-khazaka.de/index.php/en/products/ scientific/294-corneometer-2. Last Accessed May 09, 2012.

26. Fluhr JW, Kuss O, Diepgen T, Lazzerini S, Pelosi A, Gloor $M$, et al. Testing for irritation with a multifactorial approach: comparison of eight noninvasive measuring techniques on five different irritation types. $\mathrm{Br}$ J Dermatol. 2001;145(5):696-703.

27. Finlay AY, Khan GK. Dermatology Life Quality Index (DLQI) - a simple practical measure for routine clinical use. Clin Exp Dermatol. 1994;19(3):210-6.

28. Lewis-Jones MS, Finlay AY. The Children's Dermatology Life Quality Index (CDLQI): initial validation and practical use. Br J Dermatol. 1995;132(6):942-9.

29. Wolkerstorfer A, de Waard van der Spek FB, Glazenburg EJ, Mulder PG, Oranje AP. Scoring the severity of atopic dermatitis: three item severity score as a rough system for daily practice and as a pre-screening tool for studies. Acta Derm Venereol. 1999;79(5):356-9.

30. Oranje AP. Practical issues on interpretation of scoring atopic dermatitis: SCORAD Index, objective SCORAD, patient-oriented SCORAD and ThreeItem Severity score. Curr Probl Dermatol. 2011;41:149-55.

31. Hon KL, Ching GK, Leung TF, Choi CY, Lee KK, Ng PC. Estimating emollient usage in patients with eczema. Clin Exp Dermatol. 2010;35(1):22-6. 
32. Hon KL, Leung AK, Barankin B. Barrier repair therapy in atopic dermatitis: an overview. Am J Clin Dermatol. 2013;14(5):389-99.

33. Wollenberg A, Ehmann LM. Long term treatment concepts and proactive therapy for atopic eczema. Ann Dermatol. 2012;24(3):253-60.

34. Hon KL, Pong NH, Wang SS, Lee VW, Luk NM, Leung TF. Acceptability and efficacy of an emollient containing ceramide-precursor lipids and moisturizing factors for atopic dermatitis in pediatric patients. Drugs R\&D. 2013;13(1):37-42.

35. Loden $\mathrm{M}$, Andersson AC, Lindberg $\mathrm{M}$. Improvement in skin barrier function in patients with atopic dermatitis after treatment with a moisturizing cream (Canoderm). Br J Dermatol. 1999;140(2):264-7.

36. Son SW, Park SY, Ha SH, Park GM, Kim MG, Moon JS, et al. Objective evaluation for severity of atopic dermatitis by morphologic study of skin surface contours. Skin Res Technol. 2005;11(4):272-80.

37. Loden M, Andersson AC, Andersson C, Frodin T, Oman $\mathrm{H}$, Lindberg $\mathrm{M}$. Instrumental and dermatologist evaluation of the effect of glycerine and urea on dry skin in atopic dermatitis. Skin Res Technol. 2001;7(4):209-13.

38. Frosch PJKA. A method for appraising the stinging capacity of topically applied substances. J Soc Cosmet Chem. 1977;28:197-209.

39. Loden M, Andersson AC, Anderson C, Bergbrant IM, Frodin T, Ohman H, et al. A double-blind study comparing the effect of glycerin and urea on dry, eczematous skin in atopic patients. Acta Derm Venereol. 2002;82(1):45-7. 